\title{
Nuclease Delivery: Versatile Functions of SLX4/FANCP in Genome Maintenance
}

\author{
Yonghwan Kim*
}

\begin{abstract}
As a scaffold, SLX4/FANCP interacts with multiple proteins involved in genome integrity. Although not having recognizable catalytic domains, SLX4 participates in diverse genome maintenance pathways by delivering nucleases where they are needed, and promoting their cooperative execution to prevent genomic instabilities. Physiological importance of SLX4 is emphasized by the identification of causative mutations of SLX4 genes in patients diagnosed with Fanconi anemia (FA), a rare recessive genetic disorder characterized by genomic instability and predisposition to cancers. Recent progress in understanding functional roles of SLX4 has greatly expanded our knowledge in the repair of DNA interstrand crosslinks (ICLs), Holliday junction (HJ) resolution, telomere homeostasis and regulation of DNA damage response induced by replication stress. Here, these diverse functions of SLX4 are reviewed in detail.
\end{abstract}

\section{INTRODUCTION}

Human SLX4, also known as FANCP or BTBD12, is a multidomain scaffold protein composed of 1,834 amino acids with a calculated molecular mass of $200 \mathrm{kDa}$ (Fig. 1). Through direct interaction, SLX4 functions as a docking platform for the assembly of multiple genome maintenance factors including MSH2-MSH3, PLK1, TRF2, XPF-ERCC1, MUS81-EME1 and SLX1 (Fekairi et al., 2009; Munoz et al., 2009; Svendsen et al., 2009). SLX4 consists of two tandem ubiquitin-binding zinc finger (UBZ) domains, MEI9 ${ }^{\mathrm{XPF}}$ interaction like region (MLR), a Broad-complex, Tramtrack and Bric a brac (BTB), SAF-A/B, Acinus and PIAS (SAP), and SLX1 binding domain (SBD) (Fekairi et al., 2009). The UBZ domain is believed to be important for recruitment of SLX4 to the sites of ICLs (Garner and Smogorzewska, 2011; Kim and D'Andrea, 2012). The MLR, SAP

Department of Life Systems, Sookmyung Women's University, Seoul 140-742, Korea

*Correspondence: yhkim@sookmyung.ac.kr

Received 7 May, 2014; revised 27 May, 2014; accepted 28, May, 2014; published online 18 June, 2014

Keywords: fanconi anemia, Holliday junction, ICL repair, SLX4/FANCP, telomere maintenance and SBD domains are responsible for the interaction with XPFERCC1, MUS81-EME1 and SLX1, respectively (Kim et al., 2013; Wan et al., 2013). PLK1 and TRF2 bind to the middle of SLX4 (Svendsen et al., 2009). Accumulated data has proved that SLX4 plays versatile functions in maintaining genome stability by orchestrating ICL repair, $\mathrm{HJ}$ resolution, restoration of stalled replication forks and telomere maintenance, all of which require nucleolytic activities to process DNA intermediates with abnormal structures. The scaffold SLX4 has evolved to cleverly modulate these multiple processes by delivering nucleases where needed and by promoting cooperative execution to complete the processes.

SIX4 and SIx1 were initially uncovered during a synthetic lethal screen in S. cerevisiae lacking Sgs1, an ortholog of human Bloom's helicase (BLM) (Mullen et al., 2001). Orthologs of SLX1 and SLX4 have been identified and characterized in other organisms including $S$. pombe (Coulon et al., 2004), C. elegans (Saito et al., 2009), D. melanogaster (Andersen et al., 2009), mouse and human (Fig. 1). The conservation of SLX4 and SLX1 proteins among different species underscores the important physiological roles of SLX4-SLX1. Recent progress in understanding the roles of SLX4 has expanded our knowledge not only in various genome maintenance mechanisms, but also in clarifying the functions of its binding partners.

\section{ROLES OF SLX4 IN DNA INTERSTRAND CROSSLINK REPAIR}

Mutations of the SLX4/FANCP gene in Fanconi anemia $\mathrm{ICL}$ is one of the most dangerous types of DNA damage. It covalently links two strands of DNA, inhibiting transcription and replication (Deans and West, 2011). Fanconi anemia is a rare recessive genetic disease characterized by early onset of bone marrow failure, congenital abnormalities and predisposition to cancer. Cells from FA patients show enhanced sensitivity to DNA crosslinking agents. Consistently, genes mutated in FA patients are implicated in ICL repair (D'Andrea, 2010; Kottemann and Smogorzewska, 2013). The findings that human cells depleted of SLX4 are hypersensitive to DNA crosslinking agents prompted research laboratories to sequence the $S L X 4$ gene in FA patients with no known FA gene mutations. Indeed, a couple of groups identified biallelic SLX4 mutations in six patients diagnosed with FA, emphasizing the important physiological roles of SLX4 in humans (Kim et al., 2011; Stoepker et al., 2011). 


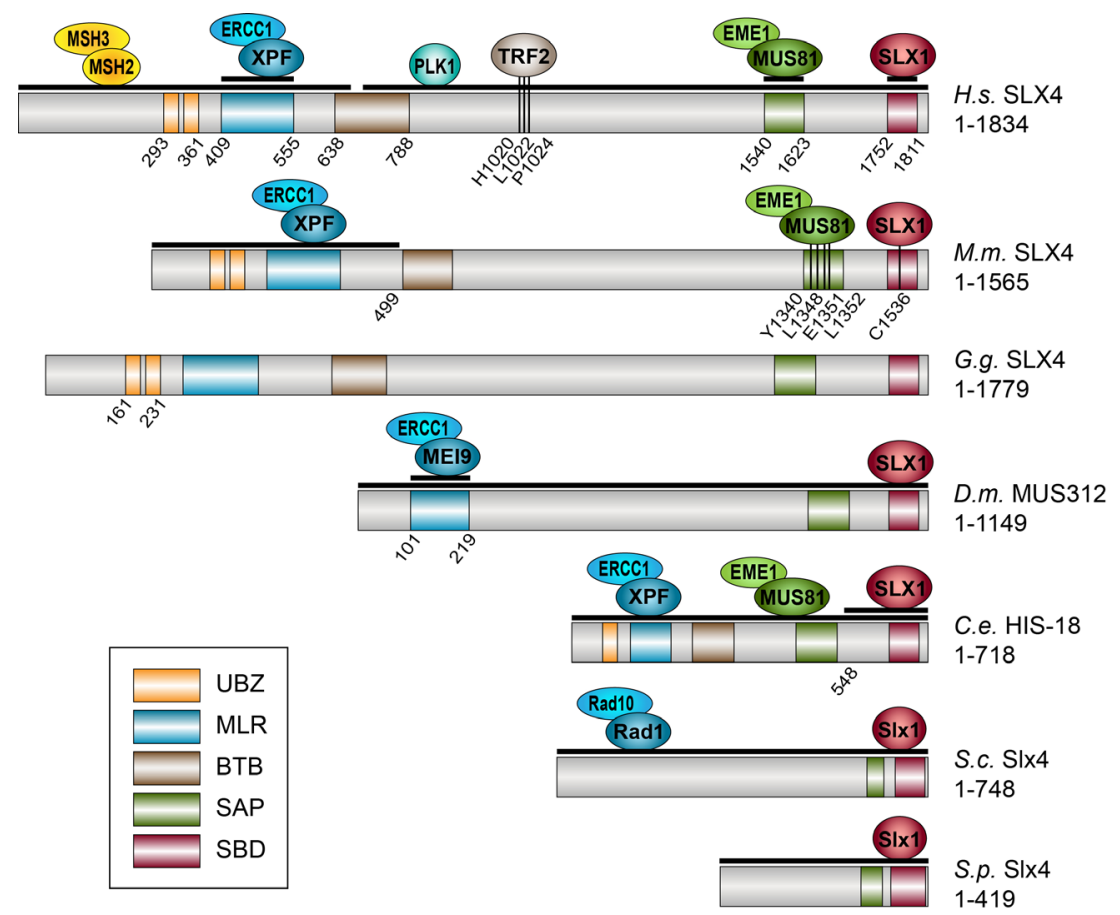

Fig. 1. Schematic comparison of domain organization and binding partners of SLX4 protein family in different species. Conserved domains are illustrated in different colors. Only reported binding partners are shown. Interacting domains are denoted by thick black lines and specific amino acid residues responsible for the interactions are indicated. The size of proteins is drawn approximately to scale. H.s., Homo sapiens; M.m., Mus musculus; G.g., Gallus gallus; D.m., Drosophila melanogaster; C.e., Caenorhabditis elegans; S.c., Saccharomyces cerevisiae; S.p., Schizosaccharomyces pombe.

UBZ domain and SLX4-XPF interaction are critical for ICL repair

In an attempt to understand the molecular mechanisms of SLX4 functions in ICL repair, a series of domain deletion mutants of SLX4 were expressed in SLX4 null human cells, showing that the UBZ domain and the XPF interacting domain (MLR) are critical for ICL repair (Kim et al., 2013) (Fig. 2A). Although the exact physiological relevance of the UBZ domain has not been determined, the UBZ domain might be important for recruitment of SLX4 to sites of ICLs (Kim and D'Andrea, 2012). In vitro ubiquitin binding showed that purified UBZ domain interacts with K-63 linked polyubiquitin chains, but not with K-48 linked polyubiquitin chains, and this interaction is abolished when the highly conserved two cysteine residues within the UBZ domain are mutated to alanine (Kim et al., 2011). In chicken DT40 cells, the UBZ domain is responsible for the interaction of SLX4 with ubiquitinated FANCD2 although this interaction has not been recapitulated in human cells (Yamamoto et al., 2011). XPF-ERCC1 is the only nuclear excision repair (NER) factor that is implicated in ICL repair (De Silva et al., 2000; Kuraoka et al., 2000). Recently, epistasis analysis showed that XPF function in ICL repair is completely dependent on SLX4 (Kim et al., 2013). Although there are conflicting views on the function of XPF in ICL repair (Bergstralh and Sekelsky, 2008), more data listed below support the idea that XPF plays a role at the incision step downstream of FANCD2, but not in homologous recombination (Castor et al., 2013; Kim et al., 2013; Kumaresan and Lambert, 2000; Kuraoka et al., 2000; Rothfuss and Grompe, 2004). First, SLX4 deficient human cells expressing SLX4 mutant that cannot interact with XPF are sen- sitive to mitomycin $\mathrm{C}$ (MMC), an ICL inducing agent, but not to camptothecin (CPT) and poly ADP ribose polymerase (PARP) inhibitor, both of which induce DNA double strand breaks (Kim et al., 2013). Consistently, HJ resolution activity of SLX4 is dependent on the interaction with MUS81 and SLX1, but not on XPF (Garner et al., 2013; Wyatt et al., 2013). Second, cells from FANCQ/XPF patients are sensitive to ICL-inducing agents, but not sensitive to CPT and PARP inhibitor although the XPF variant found in FANCQ patient still interacts with SLX4 (Bogliolo et al., 2013; Kashiyama et al., 2013). Taken together, these data support the idea that the specific function of XPF is not for homologous recombination but for other steps in the ICL repair, probably incision steps (Fig. 2A).

\section{SLX1-SLX4 is involved in ICL repair}

Depletion of SLX4 leads to a reduction in endogenous SLX1 protein level in human (Fekairi et al., 2009). Consistent with this finding, SLX1 is not detected in cells from SLX4 deficient mice. Whereas expression of wild type SLX4 in SLX $4^{-1}$ MEF restores the SLX1, expression of the SLX4 mutant that cannot interact with SLX1 fails to do so (Castor et al., 2013). Therefore, the SLX1-SLX4 interaction is essential for stability of SLX1 protein. On the contrary, SLX4 protein level is not altered in $S L X 1^{-1-}$ MEF and in human cells with siRNA-mediated SLX1 depletion. $S L X 1$ deficient mice were fertile and morphologically indistinguishable from wild type littermates. Cells from $S L X 1$ deficient mice exhibit modest sensitivity to MMC, implicating SLX1 in ICL repair. The function of SLX1 in MMC resistance requires its nuclease activity as expressing nuclease-dead SLX1 mutant in $S L X 1$ deficient MEF fails to rescue the MMC sensitivity (Castor 
A

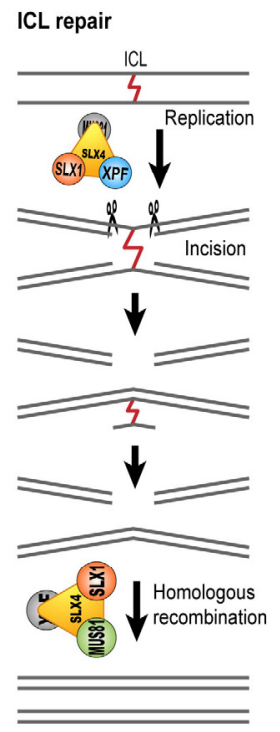

B

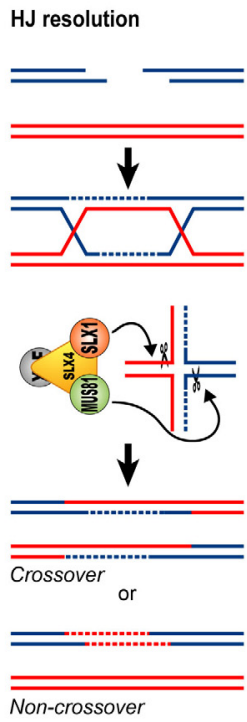

C

Telomere trimming
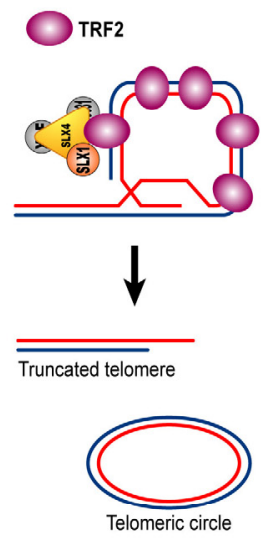

D

DNA damage response
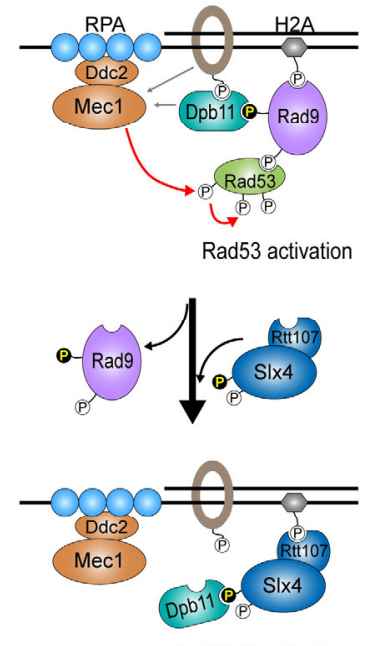

Fig. 2. Diverse functions of SLX4 in genome maintenance. For each function, participating nucleases are shown in color coded and placed in front of SLX4, whereas non-participating nucleases are depicted in gray and behind SLX4. (A) During ICL repair, SLX4 modulates XPF and potentially SLX1 in the incision step and coordinates SLX1 and MUS81 for the homologous recombination. (B) SLX4-SLX1-MUS81 functions as a $\mathrm{HJ}$ resolvase. The ordered nicking and counter-nicking transactions on HJs are mediated by SLX1 and MUS81, respectively. (C) SLX4 complex is recruited to telomeres by SLX4-TRF2 interaction and is implicated in telomere length homeostasis. SLX1 nuclease activity is important for telomere trimming. (D) Nuclease-independent function of Slx4 in budding yeast. SLX4-Rtt107 competes with Rad9 for Dpb11 and phosphorylated H2A binding, which results in down-regulation of Rad53 activity and associated DNA damage checkpoint.

et al., 2013). Since the SLX4-SLX1 complex has HJ resolvase activity, it was initially proposed that SLX1 nuclease activity plays a role in homologous recombination during the ICL repair (Munoz et al., 2009; Svendsen and Harper, 2010). However, expression of nuclease dead SLX1 fused to archaeal resolving enzyme Hje (Hje-SLX1 ${ }^{\text {mut }}$ ) in $S L X 1$ deficient MEF fails to complement MMC sensitivity, but restores Holliday junction resolvase activity. These results imply that SLX1's function in ICL repair involves cleavage of DNA intermediates rather than Holliday junctions. The target DNA intermediates of SLX1 during ICL repair remain elusive.

\section{Roles of MUS81-EME1 in ICL repair}

MUS81 deficient mice are fertile, born at normal Mendelian frequencies with no overt abnormalities (Dendouga et al., 2005) but cells from MUS81 deficient mice are sensitive to DNA crosslinking agents (Hanada et al., 2006; McPherson et al., 2004). The SAP domain of human SLX4 is responsible for SLX4MUS81 interaction, and expression of the SAP domain deletion SLX4 mutant in human SLX4 null cells partially rescue the MMC sensitivity of the FANCP cells, suggesting that the function of MUS81 in ICL repair depends on SLX4-MUS81 interaction. These findings are further supported by the results showing that depletion of MUS81 in FANCP cells results in the same MMC sensitivity as the FANCP cells. Interestingly, expression of mouse SLX4 mutants (L1348A and L1351A L1352A) that cannot interact with MUS81 were able to rescue the MMC sensitivity of SLX $4^{-/}$MEF to the same level as wildtype SLX4 (Castor et al., 2013). These findings suggest that the interaction of SLX4-MUS81 is not important for the function of MUS81 in ICL repair at least in mice, but non-SLX4-associated MUS81 might play a role in ICL repair. The SAP domain might have additional function for regulating SLX4 activities in ICL repair besides the interaction with MUS81 (Castor et al., 2013). Understanding the discrepancy of MUS81's function in ICL repair in human and mice will be interesting to study.

\section{ROLES OF SLX4 AS A HOLLIDAY JUNCTION RESOLVASE}

$\mathrm{HJ}$ is a crusade form of DNA intermediate arising at the very last step of homologous recombination during DNA double strand break repair and restoration of stalled replication forks (Liu and West, 2004). The HJ processing is required for the completion of DNA repair pathways and for chromosome segregation during mitosis ( $\mathrm{Li}$ and Heyer, 2008; Sung and Klein, 2006). In eukaryotes, $\mathrm{HJ}$ is processed either by dissolution or by resolution. The HJ dissolution is mediated by BLM-TOP3RMI1-RMI2 complex (Wu and Hickson, 2006). While molecular mechanism of $\mathrm{HJ}$ dissolution in human is relatively well understood, the resolution is not. In E. coli, the HJs are resolved by RuvC which introduces symmetrical nicks to the $\mathrm{HJ}$ to resolve it and simply religates the nicks to finish the resolution. On the other hand, SLX4-SLX1 complex introduces nicks but these nicks are not symmetric and cannot be simply ligated, and these findings raise a question if MUS81 bound to SLX4 together with SLX1 might cooperatively resolve the HJs. In eukaryotes, in vitro biochemical assays showed that three nucleases, GEN1, MUS81-EME1 and SLX4-SLX1, are capable of resolving HJs (Svendsen and Harper, 2010). Recently, physiological 
genetic relationship among the factors related to $\mathrm{HJ}$ processing was characterized.

\section{Genetic interaction of SLX4 with BLM or GEN1}

Genetic interactions of SLX4, BLM and GEN1 have been investigated using BLM deficient and SLX4 deficient human cells. Depletion of SLX4 and BLM induces cell death in BLM and SLX4 deficient cells, respectively. Further study showed that the cell death is due to severe chromosome abnormalities (Garner et al., 2013; Wyatt et al., 2013). Such abnormalities include chromosome bridges and segmented chromosomes that are observed in a large portion of cells devoid of SLX4 and BLM, leading to delayed mitotic duration and cell death. The chromosome aberrations are most likely caused by unresolved HJs linking two homologous chromosomes. Similar synthetic lethality has been observed in $S$. cerevisiae (Mullen et al., 2001), C. elegans (Saito et al., 2013) and D. melanogaster (Andersen et al., 2011) with the deletion of orthologs of BLM and SLX4 genes. Therefore, HJ processing mechanism is conserved from lower to higher eukaryotes. Depletion of MUS81 or SLX1 in BLM deficient cells also leads to cell death. Consistent with this, depletion of BLM in SLX4 null cells expressing SLX4 mutants that cannot interact with either MUS81 or SLX1 results in cell death, whereas XPF is not implicated in the synthetic lethal phenotype (Garner et al., 2013; Wyatt et al., 2013). These results suggest that among the nucleases interacting with SLX4, MUS81 and SLX1, but not XPF, are responsible for $\mathrm{HJ}$ resolution as described below.

\section{Cooperative action of SLX4-SLX1-MUS81 in HJ resolution} The MUS81-MMS4 complex has shown to be a HJ resolvase in fission yeast (Boddy et al., 2001). However, in humans, purified MUS81-EME1 does not efficiently cleave intact HJs, but does show higher resolvase activity on nicked HJs (Gaillard et al., 2003; Hollingsworth and Brill, 2004). To reconcile the genetic results and biochemical function of MUS81, it was proposed that there might be a factor that introduces a nick to intact HJs, which generates a structure that MUS81 can act on. One of the strong candidates is SLX1 as purified full length SLX4 and SLX1 complex showed a powerful nicking activity on a wide range of DNA structures including 3'-flap, 5'-flap and intact HJs. Using specific HJ substrates, Wyatt et al confirmed that SLX1 makes a nick and MUS81 finalizes HJ resolution, a sequential $\mathrm{HJ}$ resolution by two endonucleases bound to SLX4 (Wyatt et al., 2013) (Fig. 2B).

\section{ROLES OF SLX4 IN TELOMERE HOMEOSTASIS}

SLX4 is localized to telomeres through the interaction with TRF2 (Svendsen et al., 2009; Wan et al., 2013; Wilson et al., 2013). Telomere length increases when SLX4 is depleted in U2OS cells and is restored by expressing wild type SLX4. However, SLX4 mutant that cannot interact with SLX1 fails to restores telomere length, indicating that $S L X 1$ is responsible for telomere trimming (Fig. 2C). Biochemical analysis demonstrated that the endonuclease activity of SLX1 mediates the cleavage of telomeric D-loop (Wan et al., 2013). These results are reflected in vivo showing that SLX4-SLX1 is responsible for the formation of telomeric circles implying that by resolving t-loops, SLX4-SLX1 might be necessary for telomere trimming when required. It was reported that TRF2 negatively regulates the length of telomeres (Ancelin et al., 2002; Smogorzewska et al., 2000). The results that TRF2 brings SLX4 and SLX4 delivers
SLX1 to telomere shed light on how TRF2 negatively regulates the length of telomere. Intriguingly, however, the SLX4 function in telomere homeostasis is not dependent on its localization to telomeres in mice (Wilson et al., 2013). Mouse SLX4 does not contain TRF2 binding motifs and thus does not form foci at telomeres. However, it was observed that cells from SLX4 knockout mice exhibit longer telomere than wild type mice, and the longer telomere length was restored to normal when wild type SLX4 is expressed. Increased TIFs (telomere dysfunctioninduced foci) are observed in the absence of SLX4 in both human and mouse cells, indicating that SLX4 prevents DNA damage at the telomeres (Wilson et al., 2013). Understanding remains elusive of how SLX4 prevents TIF formation without localizing to telomeres in mouse. It would be interesting to study if lengthening of telomere leads to DNA damage at the telomere region as the lack of SLX4 results in longer telomere length and enhanced TIF formation. This would lead us to understand the biological relevance of telomere trimming, which is guided by TRF2-SLX4 interaction.

\section{NUCLEASES INDEPENDENT FUNCTION OF SLX4: CONTROLLING DNA DAMAGE RESPONSES}

DNA damage occurring before and during $S$ phase needs to be repaired to ensure fidelity of DNA replication. DNA insults in S phase are particularly detrimental as DNA replication machinery falls off from the DNA when it encounters unrepaired DNA damage (Cimprich and Cortez, 2008). In S. cerevisiae, Mec1 ${ }^{\text {ATR }}$ is recruited to the sites of damage, and is activated by Dpb11 ${ }^{\text {TopBP1 }}$ which independently mobilizes to DNA lesions in response to replication stress. The activated Mec1 initiates a checkpoint signaling cascade by phosphorylating multiple targets including Chk1 and Rad53. Once DNA lesions are repaired, cells need to deactivate the damage response to resume cell cycle progression. Since hyperactivated or persistent DNA damage response triggers cellular programs leading to senescence or apoptosis, the activity of kinases implicated in the processes need to be tightly regulated (Clerici et al., 2001). Recently, Ohouo et al showed that SIx4-Rtt107 complex prevents aberrant hyperactivation of DNA damage signaling induced by the DNA alkylating agent, methylmethane sulfonate. They observed that budding yeast lacking SIx4 exhibits hyperphosphorylated Rad53, indicating that SIx4 plays a role in regulating the level of Rad53 activation (Ohouo et al., 2013). The activation of the checkpoint effector Rad53 is mediated by Rad9 ${ }^{53 \mathrm{BP} 1}$ which is stabilized at the lesions through the interaction with Dpb11 and phosphorylated H2A. However, Ohouo et al found that the SIx4-Rtt107 complex occupies the Rad9 binding sites to Dpb11 and phosphorylated $\mathrm{H} 2 \mathrm{~A}$ and in turn reduces the level of Rad53 phosphorylation. Therefore, in the absence of SIx4, the checkpoint adaptor Rad9 binds to more Dpb11 and $\mathrm{H} 2 \mathrm{~A}$, and mediates more activation of Rad53 (Ohouo et al., 2013) (Fig. 2D). For the interaction between Slx4 and Dpb11, and Rtt107 and phosphorylated H2A, SIx4 and Rtt107 need to be phosphorylated by Mec1, implying that cells are evolved to fine tune the level of DNA damage response by the competition based mechanism in response to replicative stress. It is worth noting that phosphorylated SIX4 interacts with BRCT domains of Dpb11, which will be discussed later (Ohouo et al., 2010). Currently such nucleases-independent function of SIx4 has been reported only in budding yeasts; similar SLX4 function in human remains to be identified. 


\section{CONCLUSION}

Although not having enzymatic domains, SLX4 plays a critical role in preventing genomic instability by delivering multiple structure specific endonucleases to the sites of DNA lesions and coordinating their functions. One of the emerging questions to be answered is how SLX4 is recruited to different types of DNA damage. Multiple groups have provided evidence that localization of SLX4 to telomeres is mediated by SLX4-TRF2 interaction. The UBZ domain has been suggested to recruit SLX4 to sites of ICLs based on its interaction with ubiquitinated FANCD2 in chicken DT40 cells although the same interaction has not been recapitulated in mammalian cells. SLX4 could be mobilized to sites of ICLs through as of yet unidentified factors that are poly-ubiquitinated on K63 residue, for which UBZ domain has a higher affinity. The molecular mechanism of SLX4 localization to sites of DNA double strand breaks remains elusive. Based on the observation in budding yeast that phosphorylated SIx4-Rtt107 complex is targeted to stalled replication forks through the direct interaction with Dpb11, which contains BRCT domains, it would be interesting to study if ATM/ATR dependent phosphorylation of SLX4 is implicated in this localization via similar interaction with BRCT domain containing proteins. While the functional relevance of SLX4-bound nucleases, XPF-ERCC1, MUS81-EME1 and SLX1, has been relatively well understood, additional functions of SLX4 through its interaction with MSH2-MSH3, c20orf94, and PLK1 have not been characterized. In particular, as PLK1 is implicated in cell cycle dependent EME1 phosphorylation, which enhances the SLX4MUS81-EME1 interaction and $\mathrm{HJ}$ resolvase activity (Matos et al., 2011), it will be interesting to study if this function of PLK1 is dependent on its interaction with SLX4.

\section{ACKNOWLEDGMENTS}

This work was supported by the Sookmyung Women's University research grants 1-1403-0045.

\section{REFERENCES}

Ancelin, K., Brunori, M., Bauwens, S., Koering, C.E., Brun, C., Ricoul, M., Pommier, J.P., Sabatier, L., and Gilson, E. (2002). Targeting assay to study the cis functions of human telomeric proteins: evidence for inhibition of telomerase by TRF1 and for activation of telomere degradation by TRF2. Mol. Cell. Biol. 22, 3474-3487.

Andersen, S.L., Bergstralh, D.T., Kohl, K.P., LaRocque, J.R., Moore, C.B., and Sekelsky, J. (2009). Drosophila MUS312 and the vertebrate ortholog BTBD12 interact with DNA structure-specific endonucleases in DNA repair and recombination. Mol. Cell 35, 128-135.

Andersen, S.L., Kuo, H.K., Savukoski, D., Brodsky, M.H., and Sekelsky, J. (2011). Three structure-selective endonucleases are essential in the absence of BLM helicase in Drosophila. PLOS Genet. 7, e1002315.

Bergstralh, D.T., and Sekelsky, J. (2008). Interstrand crosslink repair: can XPF-ERCC1 be let off the hook? Trends Genet. 24, 70-76.

Boddy, M.N., Gaillard, P.H., McDonald, W.H., Shanahan, P., Yates, J.R., 3rd, and Russell, P. (2001). Mus81-Eme1 are essential components of a Holliday junction resolvase. Cell 107, 537-548.

Bogliolo, M., Schuster, B., Stoepker, C., Derkunt, B., Su, Y., Raams, A., Trujillo, J.P., Minguillon, J., Ramirez, M.J., Pujol, R., et al. (2013). Mutations in ERCC4, encoding the DNA-repair endonuclease XPF, cause Fanconi anemia. Am. J. Hum. Genet. 92, 800-806.

Castor, D., Nair, N., Declais, A.C., Lachaud, C., Toth, R., Macartney, T.J., Lilley, D.M., Arthur, J.S., and Rouse, J. (2013). Cooperative control of holliday junction resolution and DNA repair by the SLX1 and MUS81-EME1 nucleases. Mol. Cell 52, 221-233.
Cimprich, K.A., and Cortez, D. (2008). ATR: an essential regulator of genome integrity. Nat. Rev. Mol. Cell Biol. 9, 616-627.

Clerici, M., Paciotti, V., Baldo, V., Romano, M., Lucchini, G., and Longhese, M.P. (2001). Hyperactivation of the yeast DNA damage checkpoint by TEL1 and DDC2 overexpression. EMBO J. 20, 6485-6498

Coulon, S., Gaillard, P.H., Chahwan, C., McDonald, W.H., Yates, J.R., 3rd, and Russell, P. (2004). SIx1-SIx4 are subunits of a structure-specific endonuclease that maintains ribosomal DNA in fission yeast. Mol. Biol. Cell 15, 71-80.

D'Andrea, A.D. (2010). Susceptibility pathways in Fanconi's anemia and breast cancer. N. Engl. J. Med. 362, 1909-1919.

De Silva, I.U., McHugh, P.J., Clingen, P.H., and Hartley, J.A. (2000) Defining the roles of nucleotide excision repair and recombination in the repair of DNA interstrand cross-links in mammalian cells. Mol. Cell. Biol. 20, 7980-7990.

Deans, A.J., and West, S.C. (2011). DNA interstrand crosslink repair and cancer. Nat. Rev. Cancer 11, 467-480.

Dendouga, N., Gao, H., Moechars, D., Janicot, M., Vialard, J., and McGowan, C.H. (2005). Disruption of murine Mus81 increases genomic instability and DNA damage sensitivity but does not promote tumorigenesis. Mol. Cell. Biol. 25, 7569-7579.

Fekairi, S., Scaglione, S., Chahwan, C., Taylor, E.R., Tissier, A., Coulon, S., Dong, M.Q., Ruse, C., Yates, J.R., 3rd, Russell, P., et al. (2009). Human SLX4 is a Holliday junction resolvase subunit that binds multiple DNA repair/recombination endonucleases. Cell 138, 78-89.

Gaillard, P.H., Noguchi, E., Shanahan, P., and Russell, P. (2003). The endogenous Mus81-Eme1 complex resolves Holliday junctions by a nick and counternick mechanism. Mol. Cell 12, 747759.

Garner, E., and Smogorzewska, A. (2011). Ubiquitylation and the Fanconi anemia pathway. FEBS Lett. 585, 2853-2860.

Garner, E., Kim, Y., Lach, F.P., Kottemann, M.C., and Smogorzewska, A. (2013). Human GEN1 and the SLX4-associated nucleases MUS81 and SLX1 are essential for the resolution of replication-induced Holliday junctions. Cell Rep. 5, 207-215.

Hanada, K., Budzowska, M., Modesti, M., Maas, A., Wyman, C., Essers, J., and Kanaar, R. (2006). The structure-specific endonuclease Mus81-Eme1 promotes conversion of interstrand DNA crosslinks into double-strands breaks. EMBO J. 25, 4921-4932.

Hollingsworth, N.M., and Brill, S.J. (2004). The Mus81 solution to resolution: generating meiotic crossovers without Holliday junctions. Genes Dev. 18, 117-125.

Kashiyama, K., Nakazawa, Y., Pilz, D.T., Guo, C., Shimada, M., Sasaki, K., Fawcett, H., Wing, J.F., Lewin, S.O., Carr, L., et al. (2013). Malfunction of nuclease ERCC1-XPF results in diverse clinical manifestations and causes Cockayne syndrome, xeroderma pigmentosum, and Fanconi anemia. Am. J. Hum. Genet. 92, 807-819.

Kim, H., and D'Andrea, A.D. (2012). Regulation of DNA cross-link repair by the Fanconi anemia/BRCA pathway. Genes Dev. 26, 1393-1408.

Kim, Y., Lach, F.P., Desetty, R., Hanenberg, H., Auerbach, A.D., and Smogorzewska, A. (2011). Mutations of the SLX4 gene in Fanconi anemia. Nat. Genet. 43, 142-146.

Kim, Y., Spitz, G.S., Veturi, U., Lach, F.P., Auerbach, A.D., and Smogorzewska, A. (2013). Regulation of multiple DNA repair pathways by the Fanconi anemia protein SLX4. Blood 121, 5463.

Kottemann, M.C., and Smogorzewska, A. (2013). Fanconi anaemia and the repair of Watson and Crick DNA crosslinks. Nature 493, 356-363.

Kumaresan, K.R., and Lambert, M.W. (2000). Fanconi anemia, complementation group A, cells are defective in ability to produce incisions at sites of psoralen interstrand cross-links. Carcinogenesis 21, 741-751.

Kuraoka, I., Kobertz, W.R., Ariza, R.R., Biggerstaff, M., Essigmann, J.M., and Wood, R.D. (2000). Repair of an interstrand DNA cross-link initiated by ERCC1-XPF repair/recombination nuclease. J. Biol. Chem. 275, 26632-26636.

Li, X., and Heyer, W.D. (2008). Homologous recombination in DNA repair and DNA damage tolerance. Cell Res. 18, 99-113.

Liu, Y., and West, S.C. (2004). Happy Hollidays: 40th anniversary of the Holliday junction. Nat. Rev. Mol. Cell. Biol. 5, 937-944.

Matos, J., Blanco, M.G., Maslen, S., Skehel, J.M., and West, S.C. 
(2011). Regulatory control of the resolution of DNA recombination intermediates during meiosis and mitosis. Cell 147, 158-172.

McPherson, J.P., Lemmers, B., Chahwan, R., Pamidi, A., Migon, E., Matysiak-Zablocki, E., Moynahan, M.E., Essers, J., Hanada, K., Poonepalli, A., et al. (2004). Involvement of mammalian Mus81 in genome integrity and tumor suppression. Science 304, 18221826.

Mullen, J.R., Kaliraman, V., Ibrahim, S.S., and Brill, S.J. (2001). Requirement for three novel protein complexes in the absence of the Sgs1 DNA helicase in Saccharomyces cerevisiae. Genetics $157,103-118$.

Munoz, I.M., Hain, K., Declais, A.C., Gardiner, M., Toh, G.W., Sanchez-Pulido, L., Heuckmann, J.M., Toth, R., Macartney, T., Eppink, B., et al. (2009). Coordination of structure-specific nucleases by human SLX4/BTBD12 is required for DNA repair. Mol. Cell 35, 116-127.

Ohouo, P.Y., Bastos de Oliveira, F.M., Almeida, B.S., and Smolka, M.B. (2010). DNA damage signaling recruits the Rtt107-SIx4 scaffolds via Dpb11 to mediate replication stress response. Mol. Cell 39, 300-306.

Ohouo, P.Y., Bastos de Oliveira, F.M., Liu, Y., Ma, C.J., and Smolka, M.B. (2013). DNA-repair scaffolds dampen checkpoint signalling by counteracting the adaptor Rad9. Nature 493, 120-124.

Rothfuss, A and Grompe, M. (2004). Repair kinetics of genomic interstrand DNA cross-links: evidence for DNA double-strand break-dependent activation of the Fanconi anemia/BRCA pathway. Mol. Cell. Biol. 24, 123-134.

Saito, T.T., Youds, J.L., Boulton, S.J., and Colaiacovo, M.P. (2009). Caenorhabditis elegans HIM-18/SLX-4 interacts with SLX-1 and XPF-1 and maintains genomic integrity in the germline by processing recombination intermediates. PLoS Genet. 5, e1000735.

Saito, T.T., Lui, D.Y., Kim, H.M., Meyer, K., and Colaiacovo, M.P. (2013). Interplay between structure-specific endonucleases for crossover control during Caenorhabditis elegans meiosis. PLoS Genet. 9, e1003586.

Smogorzewska, A., van Steensel, B., Bianchi, A., Oelmann,
S.,Schaefer, M.R., Schnapp, G., and de Lange, T. (2000). Control of human telomere length by TRF1 and TRF2. Mol. Cell. Biol. 20, 1659-1668.

Stoepker, C., Hain, K., Schuster, B., Hilhorst-Hofstee, Y., Rooimans, M.A., Steltenpool, J., Oostra, A.B., Eirich, K., Korthof, E.T., Nieuwint, A.W., et al. (2011). SLX4, a coordinator of structurespecific endonucleases, is mutated in a new Fanconi anemia subtype. Nat. Genet. 43, 138-141.

Sung, P., and Klein, H. (2006). Mechanism of homologous recombination: mediators and helicases take on regulatory functions. Nat. Rev. Mol. Cell Biol. 7, 739-750.

Svendsen, J.M., and Harper, J.W. (2010). GEN1/Yen1 and the SLX4 complex: Solutions to the problem of Holliday junction resolution. Genes Dev. 24, 521-536.

Svendsen, J.M., Smogorzewska, A., Sowa, M.E., O'Connell, B.C., Gygi, S.P., Elledge, S.J., and Harper, J.W. (2009). Mammalian BTBD12/SLX4 assembles a Holliday junction resolvase and is required for DNA repair. Cell 138, 63-77.

Wan, B., Yin, J., Horvath, K., Sarkar, J., Chen, Y., Wu, J., Wan, K., Lu, J., Gu, P., Yu, E.Y., et al. (2013). SLX4 assembles a telomere maintenance toolkit by bridging multiple endonucleases with telomeres. Cell Rep. 4, 861-869.

Wilson, J.S., Tejera, A.M., Castor, D., Toth, R., Blasco, M.A., and Rouse, J. (2013). Localization-dependent and -independent roles of SLX4 in regulating telomeres. Cell Rep. 4, 853-860.

Wu, L., and Hickson, I.D. (2006). DNA helicases required for homologous recombination and repair of damaged replication forks. Ann. Rev. Genet. 40, 279-306.

Wyatt, H.D., Sarbajna, S., Matos, J., and West, S.C. (2013). Coordinated actions of SLX1-SLX4 and MUS81-EME1 for Holliday junction resolution in human cells. Mol. Cell 52, 234-247.

Yamamoto, K.N., Kobayashi, S., Tsuda, M., Kurumizaka, H., Takata, M., Kono, K., Jiricny, J., Takeda, S., and Hirota, K. (2011). Involvement of SLX4 in interstrand cross-link repair is regulated by the Fanconi anemia pathway. Proc. Natl. Acad. Sci. USA108, 6492-6496. 